\title{
Throughput Maximizing Frequency and Power Scheduling for Wireless Ad-Hoc Networks in the Low-SINR Regime
}

\author{
Nahid Saberi \\ Division of Engineering and Applied Sciences, Harvard University, Cambridge, USA. \\ Email: nahid@deas.harvard.edu
}

Received January $18^{\text {th }}, 2012$; revised February $22^{\text {nd }}, 2012$; accepted February $28^{\text {th }}, 2012$

\begin{abstract}
We study the problem of frequency and power allocation and scheduling at a time-slotted cognitive ad-hoc wireless network, where cognitive nodes share a number of frequency bands and frequency reuse is allowed. In such a network the throughput maximization problem generally results in a mixed zero-one nonlinear non-convex problem. Interestingly, in the low-SINR regime, the power allocation policy that maximizes the total throughput follows an "on/off" strategy with maximum power usage in the "on" state. In this paper we show that the on/off strategy in the low-SINR regime is also optimal with respect to throughput when scheduling users over time and frequency subject to minimum SINR requirements. We show that these additional constraints will not change the optimum strategy, but may affect the set of "on" or "off" transmitters. Also we present an approach that transforms the mixed zero-one nonlinear problem to an equivalent mixed zero-one linear problem at the expense of extra variables.
\end{abstract}

Keywords: Ad-Hoc Networks; Cognitive Networks; Optimization; Mixed Zero-One Linear Problem; Zero-One Nonlinear Problem

\section{Introduction}

We study optimal resource allocation techniques when a number of cognitive radios and primary users share the same spectral white spaces. Cognitive radios have the ability to sense the radio spectrum environment and to switch to the available frequency bands so that they do not interfere with the high priority primary users. The question of how the sensed resources should best be allocated is a fundamental question which has received a fair amount of attention in closely related ad-hoc and cognitive network communities. Depending on the type and the design objectives of the network, the optimal solution can be defined as an allocation that maximizes the total throughput in the network [1], or the one that minimizes the power consumption [2,3] while satisfying some quality of service requirements.

The problem of transmission scheduling and assigning power and frequency in a centralized fashion in order to maximize the throughput in a wireless ad hoc network where frequency reuse is allowed is a non-linear nonconvex problem. To overcome the complexity of the problem distributed algorithms [1,3-5] and approximation methods [1,4,6-10] have been introduced. To further simplify the problem the authors of [11] have focused on maximizing the number of transmissions as a measure of the throughput, and the authors of [5] considered mi- nimizing the interference instead of maximizing the throughput. In the above references communicating nodes and/or their occupied frequency bands are known in advance. The problem considered in this paper assumes that communicating nodes and their occupied frequency bands are not given and must be determined such that the total throughput of the network is maximized. We also strive on determining the optimal power allocation for a maximum throughput transmission.

In this paper we focus on centralized throughput maximization in the low-SINR regimes. In [12] we investigated a similar problem in the high-SINR regime and proposed an approach based on the concept of Geometric Programming. In the low-SINR regime, we prove that the on/off strategy maximizes the throughput under the additional constraint on the minimum SINR required for a successful transmission.

The contribution of this paper lies in suggesting a mathematical method for finding optimum solutions to the throughput maximization problem in an ad-hoc network where power, frequency and scheduling are to be determined by a central controller. This problem is approached more rigorously than in the past, where the solutions were either approximate or based on relaxing one of the scheduling, power allocation or frequency allocation requirements. 
In what follows we start by describing our assumptions and problem formulation. In Section 3 we prove that the optimal solution to our throughput maximization problem allocates either zero or maximum power to each transmitter. We also prove that the throughput maximization in low-SINR regime which is a mixed zero-one nonlinear optimization problem has an equivalent nonlinear optimization problem with real variables. We also show how our mixed zero-one non-linear problem can be transformed to an equivalent mixed zero-one linear problem. Finally, we conclude the paper in Section 4.

\section{Assumptions and Problem Formulation}

We consider a centralized cognitive network operating in the low-SINR regime composed of $N$ nodes and $K$ available frequencies, over which the communicating pairs seek to transfer data. The objective is to determine the optimal time scheduling, and frequency and power allocation such that the total throughput is maximized. We assume that Every node has access to every frequency band and a central scheduler determines which frequencies can be occupied by each cognitive pair. We also assume that frequency reuse is allowed under the condition that SINR is sufficiently large to guarantee a successful transmission. Figure 1 shows a possible frequency allocation for a network of cognitive users which have access to at most 5 frequency bands. The allocation of frequency bands is subject to a number of constraints dictated by the abilities of the transmitter and receiver technologies, as well as the physical layer protocol governing the network. We assume the following model for communication: Network is composed of $N$ cognitive nodes and $K$ available frequency bands to be used by cognitive users. The objective is to identify the best selection of cognitive transmitter-receiver pairs, allocate frequency bands to them, and define their optimal power consumptions such that the overall throughput is maximized. Frequency reuse is allowed in the network as long as the SINR is greater than a given threshold $\beta$, where $\beta$ is fixed. Each node cannot transmit and receive on the same frequency band. Every transmitter (receiver) can send to (receive from) at most one receiver (transmitter) at the same frequency the power consumption per frequency per node at each time-slot cannot exceed $P_{\max }$. The received signal at each frequency band at node $j$ is subject to additive white Gaussian noise (AWGN) of power $n_{j}$.

Based on these assumptions the joint scheduling, frequency and power allocation problem is formulated as Problem 1. All logarithms are in base 2, and equations with unspecified indices such as (3) for example, is meant to represent a set of equations which hold for all unspecified indices (such as $j=1, \cdots N$ and $k=1, \cdots K$ in (3)). The variables are defined in Table 1.

$$
\text { Maximize } \sum_{i j k} T_{i j k} \log \left(1+S I N R_{i j k}\right)
$$

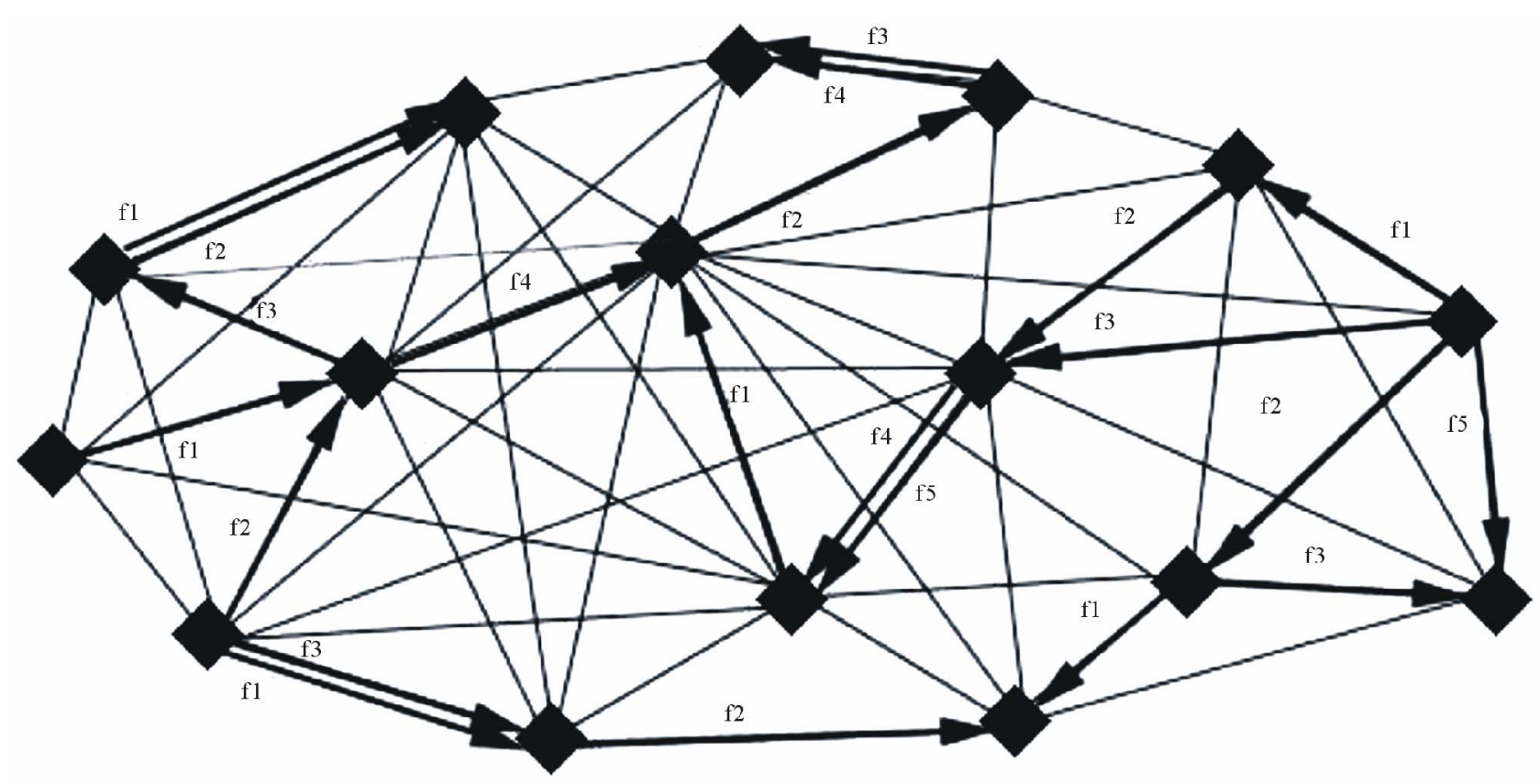

Figure 1. Visualization of a possible frequency allocation during one time-slot for an ad-hoc network of cognitive users with at most 5 available frequency bands. The arrows show a connection between a transmitter and a receiver on a specific frequency band. The narrow lines indicate a partial mesh wireless network, where a node can communicate with other nodes within its accessibility range, given the resources are available. For a node $\boldsymbol{i}$ we say a given nodes $\boldsymbol{j}$ is accessible, if $\sum_{k} \gamma_{i j k}>\mathbf{0}$. 
Table 1. Algorithms variables and parameters.

\begin{tabular}{|c|c|}
\hline Parameter/Varibale & Description \\
\hline$N$ & Number of nodes in the network \\
\hline K & Number of available frequencies \\
\hline$\gamma_{i j k}$ & Transmission gain from node $i$ to node $j$ on frequency $k$ \\
\hline$T_{i j k}$ & $\begin{array}{l}\text { Binary variable, } T_{i j k}=1 \text { if node } i \text { is transmitting to node } j \text { on frequency } k, \\
T_{i j k}=0 \text { otherwise. }\end{array}$ \\
\hline$P_{i k}$ & Amount of assigned power to transmitter $i$ on frequency $k$ \\
\hline$S I N R_{i j k}$ & $\begin{array}{l}\text { Signal to Interference plus Noise Ratio at receiver } j \text { for } \\
\text { the signal transmitted from node } i \text { on frequency } k \text {. }\end{array}$ \\
\hline$n_{j}$ & Gaussian noise at receiver $j$ \\
\hline$\beta$ & Threshold on SINR required for a successful transmission \\
\hline$A_{i j k}$ & Real variable defined as $\frac{T_{i j k}}{\sum_{m \neq i} \gamma_{m j k}\left(\sum_{\ell} T_{m \ell k}\right)+1}$ \\
\hline$B_{i m j k}$ & Real variable defined as $A_{i j k}\left(\sum T_{m e k}\right)$ \\
\hline$T T_{i m j k}$ & Binary variable defined as $T_{i j k} T_{m j k}$ \\
\hline
\end{tabular}

subject to $\operatorname{SINR}_{i j k} \geq \beta T_{i j k}$,

$\sum_{j} T_{i j k} \leq 1, \sum_{i} T_{i j k} \leq 1$,

$T_{i j k}+\sum_{\ell} T_{\ell i k} \leq 1$

$P_{i k} \leq P_{\max }$,

$T_{i j k} \in\{0,1\}, P_{i k} \in R^{+}$,

where $T_{i j k}$ is a binary variable which indicates whether or not node $i$ is transmitting to node $j$ over frequency band $k$ and $P_{i k}$ is a real variable which denotes the amount of allocated power to node $i$ on frequency $k$. If node $i$ is not transmitting to any node on frequency $k$, then $P_{i k}=0 . \operatorname{SINR}_{i j k}$ is the signal to interference plus noise ratio defined as

$$
S I N R_{i j k}=\frac{\gamma_{i j k} P_{i k}}{\sum_{m \neq i} \gamma_{m j k} P_{m k} \sum T_{m \ell k}+n_{j}},
$$

where $\gamma_{i j k}$ defines transmission gain from node $i$ to node $j$ on frequency $k$. Equation (2) corresponds to assumption 3. Equations (3) and (4) correspond to assumptions 4 and 5 for scheduling constraints. Equation (5) corresponds to assumption 6 which sets an upper bound on the power allocated to each transmitter.

\section{Scheduling and Power Control in Low-SINR Regime}

In contrast to our prior work [12], where Problem 1 was approached in the high-SINR regime, we aim to simplify the non-convex non-linear optimization problem in the low-SINR regime. This regime is particularly relevant in networks where the number of frequency bands is large and frequency reuse is common. The throughput maximization problem in this case is simplified by approximating the Shannon capacity with its first order Taylor series around zero SINR. That is, we approximate the objective function $\sum_{i j k} T_{i j k} \log \left(1+S I N R_{i j k}\right)$ at low-SINR with $\sum_{i j k} T_{i j k} S I N R_{i j k}$, keeping the remaining constraints on $T_{i j k}$ and $P_{i k}$ the same. We determine the optimum solution to this approximation to the desired problem in a number of steps:

1) In Section 3.1 we first determine a solution for the relaxation to the problem subject only to constraints on the variable domains $0 \leq T_{i j k} \leq 1$ and $0 \leq P_{i k} \leq P_{\max }$, $\forall i, j \in\{1, \cdots N\}:=I$ and $k \in\{1, \cdots, K\}:=K$.

2) Next, in Section 3.2 we determine the solution for $\boldsymbol{P}$ matrix when the minimum SINR constraints $S I N R_{i j k}>\beta T_{i j k}$ are added, $i, j \in I, k \in K$.

3) In Section 3.3 we incorporate all the scheduling constraints in (3) and (4) and show that $T_{i j k}$ will be binary using a suitable penalty function.

4) Finally, in Section 3.4 we introduce new variables which transform the non-linear optimization problem to a linear one.

The main conclusions to be drawn are that the optimal signaling strategy follows an on/off strategy, and that the optimization may be turned into an easily solvable linear program at the expense of increasing the number of 
variables from $O\left(N^{2}\right)$ to $O\left(N^{3}\right)$. The core insight follows from the work of Ebrahimi et al. [10], where the authors proved that in a similar problem without frequency allocation, if the transmission power is constrained by $P_{\max }$, the power allocation strategy which maximizes $\sum S I N R$, assigns either a power of 0 or $P_{\max }$ to each transmitter. This is called the on/off strategy. Using a similar approach we prove that the on/off strategy also maximizes the objective function of the form $\sum_{i j k} T_{i j k} S I N R_{i j k}$ subject to additional frequency and SINR constraints.

\subsection{Relaxed, Unconstrained Throughput Maximization}

We begin by removing most of the constraints from Problem 1 and relaxing the domains of $T_{i j k}$.

Theorem 1. Consider the following optimization problem:

$$
\begin{aligned}
& \text { Maximize } \sum_{i j k} T_{i j k} S I N R_{i j k} \\
& \text { subject to } 0 \leq T_{i j k} \leq 1 \\
& 0 \leq P_{i k} \leq P_{\max }
\end{aligned}
$$

1) The optimum solution to this problem allocates either 0 or $P_{\max }$ power to every node.

2) The optimum solution results in binary values for the $T_{i j k}$ variables.

Proof. A proof by contradiction was provided for the first part of the theorem by Ebrahimi et al. [10]. Using a similar argument we provide the proof for the second part. It can be seen that $H_{1}=\sum_{i j k} T_{i j k} S I N R_{i j k}$ is convex with respect to each $T_{i j k}$ since $\partial^{2} H_{1} / \partial T_{i j k}^{2} \geq 0$ (see Appendix). Thus, the maximum of $H_{1}$ will happen at the boundaries of the variable domains. To see this, assume that the optimum solution corresponds to a set $T^{*}$ with at least one non-binary element; $T_{i j k}=T_{i j k}^{*} \notin\{0,1\}$. Since $H_{1}$ is convex with respect to $T_{i j k}$, then by keeping the rest of the variables constant and moving $T_{i j \mathrm{k}}$ to the boundaries ( 0 or 1 ) one can find a larger amount for $H_{1}$ and hence the set $T^{*}$ with non-binary element cannot be the optimum solution. Therefore, $T_{i j k} \in\{0,1\}$ must hold for all $i, j, k$.

\subsection{Throughput Maximization with SINR Constraints}

The only constraint in the problem addressed in previous section are on the domains of the $P_{i k}$ and $T_{i j k}$ variables. When the SINRs are required to exceed a minimal value it is not clear if/how the optimal power allocation changes. Now we prove that adding the SINR constraint may change the set of "on" or "off" transmitters, but the on/off power allocation is still optimal. The proof is a direct result of the Generalized Lagrange Multiplier (GLM) Technique proposed by Everette [13]. Using GLM technique it can be shown that for a specific class of constrained problems, one can generate a lagrangian function of the problem such that the solution to the maximization of the lagrangian is a solution to the constrained problem. We state the needed result of [13] for clarity.

Proposition 1. Consider a constrained optimization problem of the form:

$$
\begin{aligned}
& \text { Maximize } H(x) \\
& \text { subject to } C_{k}(x) \leq c_{k}
\end{aligned}
$$

If the objective function $H(x)$ of a constrained optimization problem given by Equation (6) is concave with respect to the constraint functions $\left\{C_{k}\right\}$, then it can be guaranteed that there is a set of non-negative lagrange multipliers $\lambda_{1}, \lambda_{2}, \cdots, \lambda_{k}$ for the lagrangian of this problem, $H(x)-\sum_{k=1}^{K} \lambda_{k} C_{k}(x)$, such that the solution to the constrained optimization in Equation (6) and the solution to its lagrangian are identical [13].

We use this proposition directly in our throughput maximization to show that the on/off power strategy still holds when a constraint on each active link's SINR is enforced. Since we only define the optimum solution for $P$ matrix, we assume that the $T$ matrix is given and hence SINR is only a function of $P$. Define Problem 4:

$$
\begin{aligned}
& \text { Maximize } \sum_{i j k} T_{i j k} \operatorname{SINR}_{i j k}(P) \\
& \text { subject to } \operatorname{SINR}_{i j k}(P)>\beta T_{i j k} \\
& 0 \leq P_{i k} \leq P_{\max }
\end{aligned}
$$

We apply this Proposition to Problem 4 in the following theorem.

Theorem 2. The optimum solution to Problem 4 allocates either 0 or $P_{\max }$ power to each transmitter in the network.

Proof. To apply Proposition 1 we must show that $\mathrm{H}$ is concave with respect to $\mathrm{C}$, where $\mathrm{C}=\left[C_{i j k}\right]$. In this problem $H=\sum_{i j k} T_{i j k} \operatorname{SINR}_{i j k}(P)$ and $C_{i j k}=-\operatorname{SINR}_{i j k}(P)$. Define $S \square\left\{P: 0 \leq P_{i k} \leq P_{\max }\right\}$ the set of possible solutions for $P$. Suppose that we map every feasible matrix $P \in S$ into a point in the space of $\mathrm{H}$ and $\mathrm{C}$. Since $H=-\sum_{i j k} T_{i j k} C_{i j k}$ is a hyperplane in the $\mathrm{H}$ and $\mathrm{C}$ space, then it is both convex and concave. Note that based on our assumption $T_{i j k} \mathrm{~s}$ are constants and define the slope of the hyperplane in the $\mathrm{H}$ and $\mathrm{C}$ space. Similar to the proof of Theorem 1 it can be shown that the Lagrangian corresponding to Problem 4,

$$
\sum_{i j k} T_{i j k} \operatorname{SINR}_{i j k}+\sum_{i j k} \lambda_{i j k} \operatorname{SINR} R_{i j k},
$$

is convex with respect to every $P_{i k}$. Therefore, for any 
non-negative set of $\left\{\lambda_{i j k}\right\}$, the solution to the unconstrained Lagrangian problem follows an on/off strategy (i.e., $P_{i k} \in\left\{0, P_{\max }\right\}$ ). By Proposition 1 , the optimal solution to the unconstrained problem is equal to that of the the constrained problem, hence the solution to Problem 4 follows an on/off strategy.

\subsection{Adding Scheduling Constraints to the Optimization Problem}

The last set of constraints that must be added to the problem are the scheduling constraints which are $\sum_{i} T_{i j k} \leq 1, \quad \sum_{i} T_{i j k} \leq 1$, and $T_{i j k}+\sum_{\ell} T_{\ell i k} \leq 1$. These additional constraints violate the optimum zero-one solution for the $\boldsymbol{T}$ matrix. However, we can easily limit the solution to zero-one by adding a penalty function (i.e., $-M \sum_{i j k} T_{i j k}\left(1-T_{i j k}\right)$ to the objective function, where $M$ is a very large constant. This penalty function will take a very large value if any of the $T_{i j k} \mathrm{~s}$ has a non-binary value. Therefore the solution is directed towards a zeroone solution. Based on this discussion we state the last theorem of this section as follows:

Theorem 3. The solution to the nonlinear real-valued optimization problem defined as Problem 5:

$$
\text { Maximize } \sum_{i j k} T_{i j k} S I N R_{i j k}-M \sum_{i j k} T_{i j k}\left(1-T_{i j k}\right)
$$

subject to Equations (2)-(5)

$$
T_{i j k} \in R^{+}
$$

is a zero-one solution which is also the optimum solution to this zero-one optimization problem defined as Problem 6:

$$
\text { Maximize } \sum_{i j k} T_{i j k} S I N R_{i j k}
$$

subject to Equations (2)-(5)

$$
T_{i j k} \in\{0,1\}
$$

Proof. For large enough $M$ the optimum solution to Problem 5 is a zero-one solution, since the penalty function added to the objective function limits the solution to only zero and one values. On the other hand the solution is a maximizer for Problem 6 as it maximizes the first term in Problem 5 for binary values of $T_{i j k}$. Note that Equations (2)-(5) includes all scheduling constraints as well as the $S I N R_{i j k} \geq \beta T_{i j k}$.

Discussion. With Theorems 2 and 3 we proved that the solution to Problem 6 with zero-one variables (i.e., $T_{i j k} \in\{0,1\}$ ), is the same as the solution to Problem 5 with real variables. This significantly reduces the complexity of our problem from a mixed integer non-linear problem to a regular non-linear problem. With Theorem 2 we proved that the optimum solution to Problem 6 results in 0 or $P_{\max }$ solutions for $P_{i k}$ s. This finding helps us introduce an approach for linearizing Problem 6 in Section 3.4.

\subsection{Transforming the Throughput Maximization to a Mixed Zero-One Linear Problem}

The throughput maximization problem is a non-linear problem. However, in low-SINR regime when the transmitters follow an on/off power allocation strategy (based on Theorem 2), the problem can be transformed to a linear problem with the cost of additional variables. In the maximization problem both the objective function and the SINR constraint are non-linear. But both can be linearized using a variation of Balas method [14]. With the assumption of binary values for $T_{i j k} \mathrm{~s}$ and also 0 or $P_{\max }$ values for $P_{i k}$, the objective function $\sum_{i j k} T_{i j k} S I N R_{i j k}$ is transformed to:

$$
\sum_{i j k} \frac{\gamma_{i j k} P_{\max } T_{i j k}}{\sum_{m \neq i} \gamma_{m j k} P_{\max }\left(\sum_{\ell} T_{m \ell k}\right)+n_{j}} .
$$

Note that if $T_{i j \mathrm{k}}=1$ then $P_{i k}=P_{\max }$ and so $T_{i j k} P_{i k}=T_{i j k} P_{\max }$ holds. Also if $T_{i j k}=0$, regardless of the value of $P_{i k} \quad T_{i j k} P_{i k}=T_{i j k} P_{\max }$ holds. A similar argument can be used to explain $P_{m k} \sum_{\ell} T_{m \ell k}=P_{\max } \sum_{\ell} T_{m \ell k}$ in the denominator. Equation (7) is a non-linear function of binary variables $T_{i j \mathrm{k}}$. Without loss of generality we assume that $P_{\max }=1$ and $n_{j}=1$. Also define variables $A_{i j k} \square \frac{T_{i j k}}{\sum_{m \neq i} \gamma_{m j k}\left(\sum_{\ell} T_{m \ell k}\right)+1}$. Therefore the objective function is transformed to $\sum_{i j k} \gamma_{i j k} A_{i j k}$, with an additional constraint defining $A_{i j k}$. After crossing multiplication we obtain:

$$
\sum_{m \neq i} \gamma_{m j k}\left(\sum_{\ell} T_{m \ell k}\right) A_{i j k}+A_{i j k}=T_{i j k} .
$$

Even though $A_{i j k}$ is not a binary variable, since it is smaller than 1 , we still can use the Balas approach to linearize its multiplication with a binary variable. Note that $\sum T_{m \ell k}$ can only take values 0 or 1 due to our scheduling constraints. Let's define $B_{i m j k} \square A_{i j k}\left(\sum_{\ell} T_{m \ell k}\right)$. Then Equation (8) is transformed to a linear constraint and using Balas method we only need to add a number of linear inequalities to the problem:

$$
\begin{aligned}
& B_{i m j k} \geq 0, \\
& B_{i m j k} \leq A_{i j k}, \\
& B_{i m j k} \leq \sum_{\ell} T_{m \ell k}, \\
& B_{i m j k} \geq \sum_{\ell} T_{m \ell k}+A_{i j k}-1 .
\end{aligned}
$$

To transform the non-linear SINR constraint to a linear 
one we consider the fact that if $T_{i j k}=0$ then regardless of the value of $P_{i k}$, the constraint $\operatorname{SINR}_{i j k} \geq \beta T_{i j k}$ is satisfied. Also if $T_{i j k}=1$ then $P_{i k}=P_{\max }$ and so we can replace $P_{i k}$ in the numerator of $\operatorname{SINR}_{i j k}$ in this constraint with $P_{\max }$. We introduce additional variables $T T_{i m j k} \square T_{i j k} T_{m j k}$ to transform this constraint to a linear one. Therefore, the linear constrained optimization problem to be solved is:

$$
\text { Maximize } \sum_{i j k} \gamma_{i j k} A_{i j k}
$$

subject to

$$
\begin{aligned}
& \sum_{m \neq i} \frac{\beta \gamma_{m j k}}{\gamma_{i j k}} T T_{i m j k}+\frac{\beta}{\gamma_{i j k}} T_{i j k} \leq 1, \\
& \sum_{m \neq i} \gamma_{m j k} B_{i m j k}+A_{i j k}=T_{i j k}, \\
& \sum_{i} T_{i j k} \leq 1, \sum_{j} T_{i j k} \leq 1, \\
& 0 \leq B_{i m j k} \leq A_{i j k}, \\
& \sum_{\ell} T_{m \ell k}+A_{i j k}-1 \leq B_{i m j k} \leq \sum_{\ell} T_{m \ell k}, \\
& T T_{i m j k} \leq T_{i j k}, \\
& T_{m j k}+T_{i j k}-1 \leq T T_{i m j k} \leq T_{m j k}, \\
& T T_{i m j k}, T_{i j k} \in\{0,1\} \\
& B_{i m j k}, A_{i j k} \in R^{+}
\end{aligned}
$$

This problem is a mixed zero-one linear problem with binary variables $T T_{i m j k}, T_{i j k}$ and real variables $B_{i m j k}, A_{i j k}$. This problem can be solved using standard methods for solving mixed-integer linear problems. Note that we did not use any approximation to transform Problem 6 (nonlinear non-convex problem) to a linear one, but the cost that we pay for this transformation is an increase in the number of variables from $O\left(N^{2}\right)$ to $O\left(N^{3}\right)$. This complexity compared to the number of possible solutions in an exhaustive search which is the only reliable solution to the non-linear problems of this type is very low. The complexity of a search method for Problem 6 is $O\left(2^{N^{2}}\right)$ which is much higher than the complexity of our linear problem, especially for large values of $N$.

\section{Conclusion}

In this paper we presented methods for obtaining optimal solutions for throughput maximization in centralized cognitive networks operating in the low-SINR regime. The optimal solutions define the set of optimum communicating pairs as well as the optimum power levels and frequency allocated to the transmitters. We showed that in the low-SINR regime the mixed zero-one non-linear problem can be solved with an equivalent non-linear optimization problem. Also we proved that the optimal solution allocates $0 / P_{\max }$ powers to the transmitters. Using this result we transformed the mixed zero-one nonlinear problem to an equivalent mixed zero-one linear problem, which can be solved efficiently using the standard methods for solving mixed integer linear problems. The centralized optimum solution presented in this paper serves as a benchmarks for suggesting new distributed algorithms and evaluating approximation algorithms for both centralized and distributed methods.

\section{REFERENCES}

[1] J. Bazerque and G. Giannakis, “A Distributed Scheduling and Resource Allocation for Cognitive OFDMA Radios Bazerque," Proceedings of International IEEE Conference on Cognitive Radio Oriented Wireless Networks and Communications, Orlando, 3 August 2007, pp. 343-350.

[2] R. Cruz and A. Santhanam, “Optimal Routing, Link Scheduling and Power Control in Multi-Hop Wireless Net-Works," Proceedings of IEEE INFOCOM, San Francisco, 1-3 April 2003, pp. 702-711.

[3] G. Foschini and Z. Miljanic, "A Simple Distributed AuTonomous Power Control Algorithm and Its Convergence,” IEEE Transactions on Vehicular Technology, Vol. 42, No. 4, 1993, pp. 641-646. doi:10.1109/25.260747

[4] M. Chiang, C. W. Tan, D. Palomar, D. O’Neill and D. Julian, "Power Control by Geometric Programming," IEEE Transactions on Wireless Communications, Vol. 7, 2007, pp. 2640-2651. doi:10.1109/TWC.2007.05960

[5] B. Babadi and V. Tarokh, "A Distributed Asynchronous Algorithm for Spectrum Sharing in Wireless Ad-Hoc NetWorks," Proceedings of 42nd Annual Conference on Information Sciences and Systems, Princeton, 19-21 March 2008. doi:10.1109/CISS.2008.4558635

[6] R. Urgaonkar and M. J. Neely, “Opportunistic Scheduling with Reliability Guarantees in Cognitive Radio Networks," Proceedings of IEEE INFOCOM, Phoenix, 13-18 April 2008.

[7] Y. Y. T. Hou, Y. Shi and H. Sherali, “Optimal Spectrum Sharing for Multi-Hop Software Defined Radio Networks," Proceedings of IEEE INFOCOM, Anchorage, 6-12 May 2007.

[8] Y. Yuan, P. Bahl, R. Chandra, T. Moscibroda and Y. Wu, "Allocating Dynamic Time-Spectrum Blocks in Cognitive Radio Networks," Proceedings of IEEE/ACM MobiHoc, Montreal, 9-14 September 2007.

[9] C. Peng, H. Zheng and B. Y. Zhao, "Utilization and Fairness in Spectrum Assignment for Opportunistic Spectrum Access," ACM Mobile Networks and Applications, Vol. 11, No. 4, 2006, pp. 555-576. doi:10.1007/s11036-006-7322-y

[10] M. Ebrahimi, M. A. Maddah-Ali and A. K. Khandani, "Throughput Scaling Laws for Wireless Networks with Fading Channels," IEEE Transactions on Information Theory, Vol. 113, No. 2-3, 2007, pp. 4550-4554.

[11] J. Tang, G. Xue, C. Chandler and W. Zhang, "Link Scheduling with Power Control for Throughput Enhance- 
Ment in Multihop Wireless Networks,” IEEE Transactions on Vehicular Technology, Vol. 55, No. 3, 2006, pp. 733-742. doi:10.1109/TVT.2006.873836

[12] N. Saberi and N. Devroye, "Geometric Programming for High-SINR Resource Allocation in Wireless Ad-Hoc Networks,” IEEE Communications Letters, in Press.

[13] H. Everett, “Generalized Lagrange Multiplier Method for

\section{Appendix}

Proof of $\partial^{2} H_{1} / \partial T_{i j k}^{2} \geq 0$.

We have $H_{1}=\sum_{i j k} T_{i j k} S I N R_{i j k}$. Without loss of generality we consider a given element of matrix $T$, say $T_{12 k}$, and rewrite $H_{1}$ as:

$$
H_{1}=T_{12 k} \operatorname{SINR}_{12 k}+\sum_{i \neq 1} \sum_{j \neq 2} \sum_{k} T_{i j k} S I N R_{i j k} .
$$

The first partial differentiation is obtained as:

$$
\begin{aligned}
\partial H_{1} / \partial T_{12 k}= & \frac{\gamma_{12 k} P_{1 k}}{\sum_{m \neq 1} \gamma_{m 2 k} P_{m k} \sum_{\ell} T_{\ell m k}+n_{j}} \\
& -\sum_{i \neq 1} \sum_{j \neq 2} \sum_{k} \frac{T_{i j k} \gamma_{i j k} P_{i k} \gamma_{2 j k} P_{2 k}}{\left(\sum_{m \neq i} \gamma_{m j k} P_{m k} \sum_{\ell} T_{\ell m k}+n_{j}\right)^{2}} .
\end{aligned}
$$

The second partial differentiation is obtained as:
Solving Problems of Optimum Allocation of Resources," Operations Research, Vol. 11, No. 3, 1963, pp. 399-417. doi:10.1287/opre.11.3.399

[14] E. Balas, “An Additive Algorithm for Solving Linear ProGrams with Zero-One Variables,” Operations Research, Vol. 13, No. 4, 1965, pp. 517-546.

$$
\begin{aligned}
\partial^{2} H_{1} / \partial T_{12 k}^{2}= & \frac{-\gamma_{12 k} P_{1 k} \gamma_{22 k} P_{2 k}}{\left(\sum_{m \neq 1} \gamma_{m 2 k} P_{m k} \sum_{\ell} T_{\ell m k}+n_{j}\right)^{2}} \\
& +\sum_{i \neq 1} \sum_{j \neq 2} \sum_{k} \frac{T_{i j k} \gamma_{i j k} P_{i k} \gamma_{2 j k}^{2} P_{2 k}^{2}}{\left(\sum_{m \neq i} \gamma_{m j k} P_{m k} \sum_{\ell} T_{\ell m k}+n_{j}\right)^{3}} .
\end{aligned}
$$

Since $\gamma_{22 k}=0$, the first term in the last equation is zero, and the second term is always positive. Therefore, $\partial^{2} H_{1} / \partial T_{i j k}^{2} \geq 0$ always holds. 